\title{
DES14X3taz: A TYPE I SUPERLUMINOUS SUPERNOVA SHOWING A LUMINOUS, RAPIDLY COOLING INITIAL PRE-PEAK BUMP
}

M. Smith ${ }^{1}$, M. Sullivan ${ }^{1}$, C. B. D’Andrea ${ }^{1,2}$, F. J. Castander ${ }^{3}$, R. Casas ${ }^{3}$, S. Prajs ${ }^{1}$, A. Papadopoulos ${ }^{2,4}$, R. C. Nichol $^{2}$, N. V. Karpenka ${ }^{1}$, S. R. Bernard ${ }^{5}$, P. Brown ${ }^{6}$, R. Cartier ${ }^{1}$, J. Cooke ${ }^{7}$, C. Curtin ${ }^{7}$, T. M. Davis ${ }^{8,9}$, D. A. Finley ${ }^{10}$, R. J. Foley ${ }^{11,12}$, A. Gal-Yam ${ }^{13}$, D. A. Goldstein ${ }^{14,15}$, S. GonZÁlez-Gaitán ${ }^{16,17}$, R. R. Gupta ${ }^{18}$, D. A. Howell ${ }^{19,20}$,

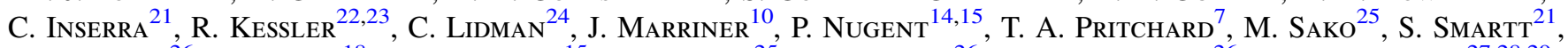
R. C. Smith $^{26}$, H. Spinka ${ }^{18}$, R. C. Thomas ${ }^{15}$, R. C. Wolf ${ }^{25}$, A. Zenteno ${ }^{26}$, T. M. C. Abbotr ${ }^{26}$, A. Benoit-Lévy ${ }^{27,28,29}$, E. Bertin ${ }^{27,29}$, D. Brooks ${ }^{28}$, E. Buckley-Geer ${ }^{10}$, A. Carnero Rosell ${ }^{30,31}$, M. Carrasco Kind ${ }^{11,32}$, J. Carretero ${ }^{3,33}$, M. Crocce $^{3}$, C. E. Cunha ${ }^{34}$, L. N. Da Costa ${ }^{30,31}$, S. Desai ${ }^{35,36}$, H. T. Diehl ${ }^{10}$, P. Doel ${ }^{28}$, J. Estrada ${ }^{10}$, A. E. Evrard ${ }^{37,38}$, B. Flaugher ${ }^{10}$, P. Fosalba ${ }^{3}$, J. Frieman ${ }^{10,22}$, D. W. Gerdes ${ }^{38}$, D. Gruen ${ }^{34,39,40,41}$, R. A. GruendL ${ }^{11,32}$, D. J. James ${ }^{26}$, K. Kuehn ${ }^{24}$, N. Kuropatkin ${ }^{10}$, O. Lahav ${ }^{28}$, T. S. Li ${ }^{42}$, J. L. Marshall ${ }^{42}$, P. Martini ${ }^{43,44}$, C. J. Miller ${ }^{37,38}$, R. Miquel ${ }^{33,45}$, B. Nord ${ }^{10}$, R. Ogando ${ }^{30,31}$, A. A. Plazas ${ }^{46}$, K. Reil ${ }^{40}$, A. K. Romer ${ }^{47}$, A. Roodman ${ }^{34,40}$, E. S. RYKOFF ${ }^{34,40}$, E. SANCHEZ ${ }^{48}$, V. Scarpine ${ }^{10}$, M. Schubnell ${ }^{38}$, I. Sevilla-Noarbe ${ }^{11,48}$, M. Soares-Santos ${ }^{10}$, F. Sobreira ${ }^{10,30}$, E. Suchyta ${ }^{25}$, M. E. C. Swanson ${ }^{32}$, G. TARLE ${ }^{38}$, A. R. Walker ${ }^{26}$, AND W. Wester ${ }^{10}$

(The DES Collaboration)

${ }^{1}$ School of Physics and Astronomy, University of Southampton, Southampton SO17 1BJ, UK; mat.smith@ soton.ac.uk 2 Institute of Cosmology \& Gravitation, University of Portsmouth, Portsmouth PO1 3FX, UK

${ }^{3}$ Institut de Ciències de l'Espai, IEEC-CSIC, Campus UAB, Carrer de Can Magrans, s/n, E-08193 Bellaterra, Barcelona, Spain

${ }^{4}$ School of Sciences, European University Cyprus, 6 Diogenes Street, Engomi, 1516 Nicosia, Cyprus

5 School of Physics, University of Melbourne, Parkville, VIC 3010, Australia

${ }^{6}$ George P. and Cynthia Woods Mitchell Institute for Fundamental Physics \& Astronomy, Department of Physics and Astronomy, Texas A\&M University, 4242 TAMU, College Station, TX 77843, USA

${ }^{7}$ Centre for Astrophysics \& Supercomputing, Swinburne University of Technology, Hawthorn, VIC 3122, Australia

${ }^{8}$ School of Mathematics and Physics, University of Queensland, Brisbane, QLD 4072, Australia

${ }^{9}$ ARC Centre of Excellence for All-sky Astrophysics (CAASTRO), Australia

${ }^{10}$ Fermi National Accelerator Laboratory, P.O. Box 500, Batavia, IL 60510, USA

${ }^{11}$ Department of Astronomy, University of Illinois, 1002 W. Green Street, Urbana, IL 61801, USA

12 Department of Physics, University of Illinois, 1110 W. Green Street, Urbana, IL 61801, USA

${ }^{13}$ Department of Particle Physics and Astrophysics, Weizmann Institute of Science, Rehovot 76100, Israel

${ }_{15}^{14}$ Astronomy Department, University of California at Berkeley, Berkeley, CA 94720, USA

${ }^{15}$ Lawrence Berkeley National Laboratory, 1 Cyclotron Road, Berkeley, CA 94720, USA

${ }^{16}$ Millennium Institute of Astrophysics, Casilla 36-D, Santiago, Chile

${ }^{17}$ Centro de Modelamiento Matemtico, Universidad de Chile, Beaucheff 851, edificio norte, piso 7, Santiago, Chile

${ }_{18}$ Argonne National Laboratory, 9700 South Cass Avenue, Lemont, IL 60439, USA

${ }^{19}$ Las Cumbres Observatory Global Telescope Network, Goleta, CA 93117, USA

${ }^{20}$ Department of Physics, University of California, Santa Barbara, CA 93106-9530, USA

${ }^{21}$ Astrophysics Research Centre, School of Mathematics and Physics, Queen's University Belfast, Belfast BT7 1NN, UK

${ }^{22}$ Kavli Institute for Cosmological Physics, University of Chicago, Chicago, IL 60637, USA

${ }^{23}$ Department of Physics and Astronomy, 5640 South Ellis Avenue, University of Chicago, Chicago, IL 60637, USA ${ }^{24}$ Australian Astronomical Observatory, North Ryde, NSW 2113, Australia

${ }^{25}$ Department of Physics and Astronomy, University of Pennsylvania, Philadelphia, PA 19104, USA

${ }^{26}$ Cerro Tololo Inter-American Observatory, National Optical Astronomy Observatory, Casilla 603, La Serena, Chile ${ }^{27}$ CNRS, UMR 7095, Institut d'Astrophysique de Paris, F-75014 Paris, France

${ }^{28}$ Department of Physics \& Astronomy, University College London, Gower Street, London WC1E 6BT, UK

${ }^{29}$ Sorbonne Universités, UPMC Univ Paris 06, UMR 7095, Institut d'Astrophysique de Paris, F-75014 Paris, France

${ }^{30}$ Laboratório Interinstitucional de e-Astronomia-LIneA, Rua Gal. José Cristino 77, Rio de Janeiro, RJ-20921-400, Brazil

${ }^{31}$ Observatório Nacional, Rua Gal. José Cristino 77, Rio de Janeiro, RJ-20921-400, Brazil

${ }^{32}$ National Center for Supercomputing Applications, 1205 West Clark Street, Urbana, IL 61801, USA

${ }^{33}$ Institut de Física d'Altes Energies (IFAE), The Barcelona Institute of Science and Technology, Campus UAB, E-08193 Bellaterra (Barcelona), Spain

${ }^{34}$ Kavli Institute for Particle Astrophysics \& Cosmology, P.O. Box 2450, Stanford University, Stanford, CA 94305, USA

${ }^{35}$ Excellence Cluster universe, Boltzmannstr. 2, D-85748 Garching, Germany

${ }^{36}$ Faculty of Physics, Ludwig-Maximilians University, Scheinerstr. 1, D-81679 Munich, Germany

${ }^{37}$ Department of Astronomy, University of Michigan, Ann Arbor, MI 48109, USA

${ }^{38}$ Department of Physics, University of Michigan, Ann Arbor, MI 48109, USA

${ }^{39}$ Max Planck Institute for Extraterrestrial Physics, Giessenbachstrasse, D-85748 Garching, Germany

${ }^{40}$ SLAC National Accelerator Laboratory, Menlo Park, CA 94025, USA

${ }^{41}$ Universitäts-Sternwarte, Fakultät für Physik, Ludwig-Maximilians Universität München, Scheinerstrasse 1, D-81679 München, Germany

${ }^{42}$ George P. and Cynthia Woods Mitchell Institute for Fundamental Physics and Astronomy, and Department of Physics and Astronomy, Texas A\&M University, College Station, TX 77843, USA

${ }^{43}$ Center for Cosmology and Astro-Particle Physics, The Ohio State University, Columbus, OH 43210, USA

${ }_{44}$ Department of Astronomy, The Ohio State University, Columbus, OH 43210, USA

${ }^{45}$ Institució Catalana de Recerca i Estudis Avançats, E-08010 Barcelona, Spain
Jet Propulsion Laboratory, California Institute of Technology, 4800 Oak Grove Drive, Pasadena, CA 91109, USA

${ }_{48}$ Department of Physics and Astronomy, Pevensey Building, University of Sussex, Brighton BN1 9QH, UK

${ }^{48}$ Centro de Investigaciones Energéticas, Medioambientales y Tecnológicas (CIEMAT), Madrid, Spain

Received 2015 December 15; accepted 2016 January 19; published 2016 February 3 


\section{ABSTRACT}

We present DES14X3taz, a new hydrogen-poor superluminous supernova (SLSN-I) discovered by the Dark Energy Survey (DES) supernova program, with additional photometric data provided by the Survey Using DECam for Superluminous Supernovae. Spectra obtained using Optical System for Imaging and low-IntermediateResolution Integrated Spectroscopy on the Gran Telescopio CANARIAS show DES14X3taz is an SLSN-I at $z=0.608$. Multi-color photometry reveals a double-peaked light curve: a blue and relatively bright initial peak that fades rapidly prior to the slower rise of the main light curve. Our multi-color photometry allows us, for the first time, to show that the initial peak cools from 22,000 to $8000 \mathrm{~K}$ over 15 rest-frame days, and is faster and brighter than any published core-collapse supernova, reaching $30 \%$ of the bolometric luminosity of the main peak. No physical ${ }^{56} \mathrm{Ni}$-powered model can fit this initial peak. We show that a shock-cooling model followed by a magnetar driving the second phase of the light curve can adequately explain the entire light curve of DES14X3taz. Models involving the shock-cooling of extended circumstellar material at a distance of $\simeq 400 R_{\odot}$ are preferred over the cooling of shock-heated surface layers of a stellar envelope. We compare DES14X3taz to the few double-peaked SLSN-I events in the literature. Although the rise times and characteristics of these initial peaks differ, there exists the tantalizing possibility that they can be explained by one physical interpretation.

Key words: supernovae: general

\section{INTRODUCTION}

Over the last 10 years, wide-field optical surveys have uncovered a new class of highly luminous transients: "superluminous" supernovae (SLSNe; see review of Gal-Yam 2012). At $M_{\text {peak }}<-21 \mathrm{mag}$, SLSNe are 10-100 times brighter than classical core-collapse SN events, but are rarer, with $<0.1 \%$ of the rate (Quimby et al. 2013; McCrum et al. 2015; S. Prajs et al. 2016, in preparation) and only 30 well-studied examples. A physical understanding of these extreme events is still emerging. There are at least two distinct classes (GalYam 2012): SLSNe-II show (narrow) hydrogen emission lines, believed to be generated by interaction with circumstellar material (CSM; Ofek et al. 2007; Smith et al. 2007; Gezari et al. 2009; Benetti et al. 2014), whereas SLSNe-I are hydrogen poor (Quimby et al. 2011), and would traditionally be classified as type Ic SNe. SLSNe-I almost invariably explode in galaxies that are low-mass, compact dwarfs (Neill et al. 2011; Lunnan et al. 2015) and that are metal-poor and strongly star-forming (Chen et al. 2013; Lunnan et al. 2013; Leloudas et al. 2015).

The power source of classical SNe Ic, the radioactive decay of ${ }^{56} \mathrm{Ni}$, cannot easily explain SLSNe-I: several solar masses of ${ }^{56} \mathrm{Ni}$ are required to reach $M_{\text {peak }}<-21$, and the light curves are difficult to reproduce with a model that has an ejecta mass greater than the ${ }^{56} \mathrm{Ni}$ mass $\left(M_{\mathrm{ej}}>M_{\mathrm{Ni}}\right.$; e.g., Chatzopoulos et al. 2009; Chomiuk et al. 2011; Inserra et al. 2013; Papadopoulos et al. 2015). Alternative power sources have been proposed, the most popular of which is energy input from a central engine, such as the spin-down of a newly formed and rapidly rotating magnetar (Kasen \& Bildsten 2010; Woosley 2010) or accretion of fallback material onto a compact object (Dexter \& Kasen 2013). Interactions between SN ejecta and a hydrogen-deficient CSM have also been studied (Chevalier \& Irwin 2011; Chatzopoulos et al. 2013).

A new puzzle has now emerged: some SLSNe-I have double-peaked light curves, with an early initial peak a few days after the inferred explosion epoch (Nicholl \& Smartt 2016). Two events in the literature have particularly prominent examples: SN2006oz (Leloudas et al. 2012) and LSQ14bdq (Nicholl et al. 2015). In both events, the initial peak lasts a few days in the rest-frame. Nicholl et al. (2015) modeled the initial peak of LSQ14bdq and found it inconsistent with being powered by ${ }^{56} \mathrm{Ni}$, favoring instead luminosity from the shockheated surface layers of the exploding star (e.g., Nakar \& Sari
2010; Rabinak \& Waxman 2011), followed by reheating by a central engine to drive the second peak. In this model, the brightness and duration of the LSQ14bdq initial peak would imply a progenitor radius of a few hundred solar radii. Other explanations that can also produce double-peaked light curves include shock heating of much more extended material (Piro 2015), or a shock breakout driven by a magnetar (Kasen et al. 2015).

Here, we present DES14X3taz, a double-peaked SLSN-I discovered by the Dark Energy Survey (DES; Flaugher et al. 2015) supernova (SN) program (DES-SN). Lying at a redshift $z=0.608$, the DES-SN griz light curves probe restframe 3000-6000 $\AA$ and provide unique multi-color information of the initial peak. These multi-color data allow us, for the first time, to estimate the temperature and temperature evolution of the initial peak for comparison with various models. We assume $H_{0}=70 \mathrm{~km} \mathrm{~s}^{-1} \mathrm{Mpc}^{-1}$ and a flat $\Lambda \mathrm{CDM}$ cosmology with $\Omega_{\text {matter }}=0.3$ throughout.

\section{OBSERVATIONS}

DES14X3taz was discovered as a transient event at $r \simeq 23.0$ by DES-SN in DECam (Flaugher et al. 2015) griz images taken on 2014 December 21.1 (all dates UTC) at $\alpha=02^{\mathrm{h}} 28^{\mathrm{m}} 04^{\mathrm{s}} .46$, $\delta=-04^{\circ} 05^{\prime} 12$ !! 7 (J2000), with previous non-detections on 2014 December 14.1. The field was imaged again, and DES14X3taz was designated as a transient object on 2014 December 27.1. As the light curve was monitored in griz by DES over the next 2-3 weeks, the existence of a pronounced initial peak in the light curve became clear, and the object was prioritized for spectroscopic observations. DES monitored DES14X3taz approximately weekly until 2015 February, and further light curve data were then obtained via the Search Using DECam for Superluminous Supernova (SUDSS) project in 2015 March and again in 2015 July. DES re-commenced 2015 August, with DES14X3taz still detected in the data. Further details of the DES-SN difference-imaging search pipeline can be found in Kessler et al. (2015) and Goldstein et al. (2015), with SLSN-specific details in Papadopoulos et al. (2015). Photometric measurements were made on the DES-SN and SUDSS data using the same pipeline as in Papadopoulos et al. (2015), which has also been used extensively in the literature (e.g., Firth et al. 2015 and references therein). The light curve data are presented in Table 1 and Figure 1; they are 
Table 1

Light Curve Data for DES14X3taz

\begin{tabular}{|c|c|c|c|c|c|c|}
\hline $\begin{array}{l}\text { Calendar } \\
\text { Date }\end{array}$ & MJD & $\begin{array}{l}\text { Phase } \\
\text { (days) }^{\mathrm{a}}\end{array}$ & $\begin{array}{c}f_{g} \\
\text { (counts) }^{\mathrm{b}}\end{array}$ & $\begin{array}{c}f_{r} \\
\text { (counts) }\end{array}$ & $\begin{array}{c}f_{i} \\
\text { (counts) }\end{array}$ & $\begin{array}{c}f_{z} \\
\text { (counts) }\end{array}$ \\
\hline 2014 Dec 10 & 57001.1 & -49.1 & $-69.0 \pm 81.1$ & $-56.9 \pm 55.8$ & $0.3 \pm 45.2$ & $-7.9 \pm 67.6$ \\
\hline 2014 Dec 14 & 57005.1 & -46.6 & $-23.3 \pm 68.1$ & $4.3 \pm 45.8$ & $-5.6 \pm 61.7$ & $\ldots$ \\
\hline 2014 Dec 14 & 57005.2 & -46.5 & $\ldots$ & $\ldots$ & $\ldots$ & $28.6 \pm 90.4$ \\
\hline 2014 Dec 21 & 57012.1 & -42.2 & $1914.4 \pm 61.4$ & $1531.4 \pm 41.8$ & $1203.2 \pm 58.5$ & $\ldots$ \\
\hline 2014 Dec 21 & 57012.2 & -42.2 & $\ldots$ & $\ldots$ & $\ldots$ & $988.7 \pm 120.9$ \\
\hline 2014 Dec 27 & 57018.1 & -38.5 & $1973.4 \pm 70.1$ & $1672.0 \pm 58.5$ & $\ldots$ & $\ldots$ \\
\hline 2014 Dec 27 & 57018.2 & -38.5 & $\ldots$ & $\ldots$ & $1792.7 \pm 87.0$ & $\ldots$ \\
\hline 2014 Dec 28 & 57019.1 & -37.9 & $\ldots$ & $\ldots$ & $\ldots$ & $1391.8 \pm 82.6$ \\
\hline 2015 Jan 03 & 57025.1 & -34.1 & $\ldots$ & $1444.2 \pm 118.3$ & $1393.4 \pm 104.0$ & $\ldots$ \\
\hline 2015 Jan 04 & 57026.1 & -33.5 & $\ldots$ & $\ldots$ & $\ldots$ & $1152.0 \pm 88.3$ \\
\hline 2015 Jan 07 & 57029.1 & -31.7 & $438.4 \pm 120.5$ & $\ldots$ & $\ldots$ & $\ldots$ \\
\hline 2015 Jan 10 & 57032.1 & -29.8 & $\ldots$ & $1779.6 \pm 83.4$ & $1958.4 \pm 69.7$ & $\ldots$ \\
\hline 2015 Jan 11 & 57033.1 & -29.2 & $\ldots$ & $\ldots$ & $\ldots$ & $2084.6 \pm 77.9$ \\
\hline 2015 Jan 14 & 57036.0 & -27.3 & $1591.4 \pm 59.6$ & $\ldots$ & $\ldots$ & $\ldots$ \\
\hline 2015 Jan 16 & 57038.1 & -26.0 & $\ldots$ & $3308.9 \pm 44.5$ & $\ldots$ & $\ldots$ \\
\hline 2015 Jan 17 & 57039.1 & -25.5 & $\ldots$ & $\ldots$ & $3536.7 \pm 63.8$ & $\ldots$ \\
\hline 2015 Jan 18 & 57040.1 & -24.8 & $\ldots$ & $\ldots$ & $\ldots$ & $3297.5 \pm 70.5$ \\
\hline 2015 Jan 20 & 57042.1 & -23.6 & $3441.5 \pm 71.8$ & $\ldots$ & $\ldots$ & $\ldots$ \\
\hline 2015 Jan 22 & 57044.1 & -22.3 & $\ldots$ & $5290.8 \pm 113.9$ & $5195.1 \pm 91.5$ & $\ldots$ \\
\hline 2015 Jan 23 & 57045.1 & -21.7 & $\ldots$ & $\ldots$ & $\ldots$ & $4753.5 \pm 110.3$ \\
\hline 2015 Jan 27 & 57049.1 & -19.2 & $5757.2 \pm 195.3$ & $\ldots$ & $\ldots$ & $\ldots$ \\
\hline 2015 Jan 29 & 57051.1 & -18.0 & $\ldots$ & $7849.3 \pm 85.4$ & $7481.1 \pm 85.2$ & $\ldots$ \\
\hline 2015 Jan 30 & 57052.1 & -17.4 & $\ldots$ & $\ldots$ & $\ldots$ & $6690.9 \pm 72.0$ \\
\hline 2015 Feb 07 & 57060.0 & -12.4 & $9105.5 \pm 100.7$ & $11139.1 \pm 97.2$ & $10361.2 \pm 89.6$ & $\ldots$ \\
\hline 2015 Feb 07 & 57060.1 & -12.4 & $\ldots$ & $\ldots$ & $\ldots$ & $9206.2 \pm 134.1$ \\
\hline 2015 Feb 25 & 57078.0 & -1.2 & $11068.6 \pm 342.2$ & $14419.4 \pm 376.7$ & $14160.5 \pm 263.2$ & $12316.9 \pm 321.3$ \\
\hline 2015 Mar 11 & 57092.0 & 7.5 & $10294.3 \pm 574.2$ & $15273.9 \pm 389.2$ & $\ldots$ & $\ldots$ \\
\hline 2015 Jul 08 & 57211.4 & 81.7 & $775.2 \pm 394.8$ & $552.2 \pm 318.1$ & $1085.7 \pm 247.7$ & $2179.9 \pm 201.8$ \\
\hline 2015 Jul 25 & 57228.3 & 92.3 & $\ldots$ & $\ldots$ & $1463.7 \pm 133.5$ & $1762.1 \pm 201.5$ \\
\hline 2015 Jul 25 & 57228.4 & 92.3 & $165.7 \pm 76.4$ & $438.1 \pm 77.0$ & $\ldots$ & $\ldots$ \\
\hline 2015 Aug 15 & 57249.3 & 105.3 & $224.0 \pm 48.5$ & $377.7 \pm 39.1$ & $714.3 \pm 45.7$ & $\ldots$ \\
\hline 2015 Aug 15 & 57249.4 & 105.3 & $\ldots$ & $\ldots$ & $\ldots$ & $940.0 \pm 52.7$ \\
\hline 2015 Aug 19 & 57253.4 & 107.8 & $66.8 \pm 39.5$ & $284.5 \pm 33.6$ & $633.2 \pm 43.3$ & $\ldots$ \\
\hline 2015 Aug 21 & 57255.3 & 109.0 & $\ldots$ & $\ldots$ & $\ldots$ & $884.0 \pm 75.8$ \\
\hline 2015 Aug 23 & 57257.3 & 110.3 & $96.5 \pm 39.4$ & $282.6 \pm 32.2$ & $\ldots$ & $\ldots$ \\
\hline 2015 Aug 23 & 57257.4 & 110.3 & $\ldots$ & $\ldots$ & $646.9 \pm 34.3$ & $\ldots$ \\
\hline 2015 Aug 31 & 57265.3 & 115.2 & $\cdots$ & $\cdots$ & $\ldots$ & $738.9 \pm 102.5$ \\
\hline 2015 Aug 31 & 57265.4 & 115.3 & $\cdots$ & $\ldots$ & $702.4 \pm 96.1$ & $\ldots$ \\
\hline 2015 Sep 03 & 57268.2 & 117.1 & $\ldots$ & $160.4 \pm 186.7$ & $\ldots$ & $\cdots$ \\
\hline 2015 Sep 04 & 57269.2 & 117.7 & $245.0 \pm 188.4$ & $\ldots$ & $\ldots$ & $\ldots$ \\
\hline 2015 Sep 08 & 57273.4 & 120.2 & $\ldots$ & $\ldots$ & $525.8 \pm 58.8$ & $879.2 \pm 69.8$ \\
\hline 2015 Sep 13 & 57278.2 & 123.3 & $\ldots$ & $328.2 \pm 47.3$ & $\ldots$ & $\ldots$ \\
\hline 2015 Sep 13 & 57278.3 & 123.3 & $89.6 \pm 50.3$ & $\ldots$ & $\ldots$ & $\ldots$ \\
\hline 2015 Sep 18 & 57283.2 & 126.4 & $\ldots$ & $\ldots$ & $363.2 \pm 58.9$ & $782.6 \pm 54.2$ \\
\hline 2015 Sep 22 & 57287.2 & 128.8 & $452.9 \pm 210.8$ & $178.4 \pm 63.9$ & $\ldots$ & $\ldots$ \\
\hline 2015 Sep 22 & 57287.3 & 128.9 & $\ldots$ & $\ldots$ & $470.6 \pm 76.0$ & $678.8 \pm 87.6$ \\
\hline
\end{tabular}

Notes.

a Relative to maximum light in the rest-frame.

${ }^{\mathrm{b}}$ Fluxes $f$ in each filter are given in counts. A zeropoint of 31.0 converts counts into AB magnitudes.

also available from the WISeREP $\operatorname{archive}^{49}$ (Yaron \& GalYam 2012).

DES14X3taz was spectroscopically observed on 2015 January 26 (when the target was $r \simeq 21.5$ ) at the Gran Telescopio CANARIAS (GTC) using the Optical System for Imaging and low-Intermediate-Resolution Integrated Spectroscopy and the R500R grism. The spectrum was taken in relatively poor conditions (bright sky, 1 "' 1 seeing) and is of low signal-to-noise ratio, so it was re-observed on 2015 February 6

49 http://wiserep.weizmann.ac.il/ in dark conditions and 0 ". 8 seeing. The spectra were reduced using standard Image Reduction and Analysis Facility (IRAF) routines and have an effective wavelength coverage of $5200-9000 \AA$ (observer frame). The spectra are spectroscopically similar and available from WISeREP.

Figure 2 shows the spectrum obtained on 2015 February 6. Weak $\mathrm{H} \beta$, [O II], and [O III] host-galaxy emission lines give a redshift of $z=0.608$. A comparison with literature spectra using SUPERFIT (Howell et al. 2005) gives an excellent match to a hydrogen-poor SLSN. The broad absorption features 

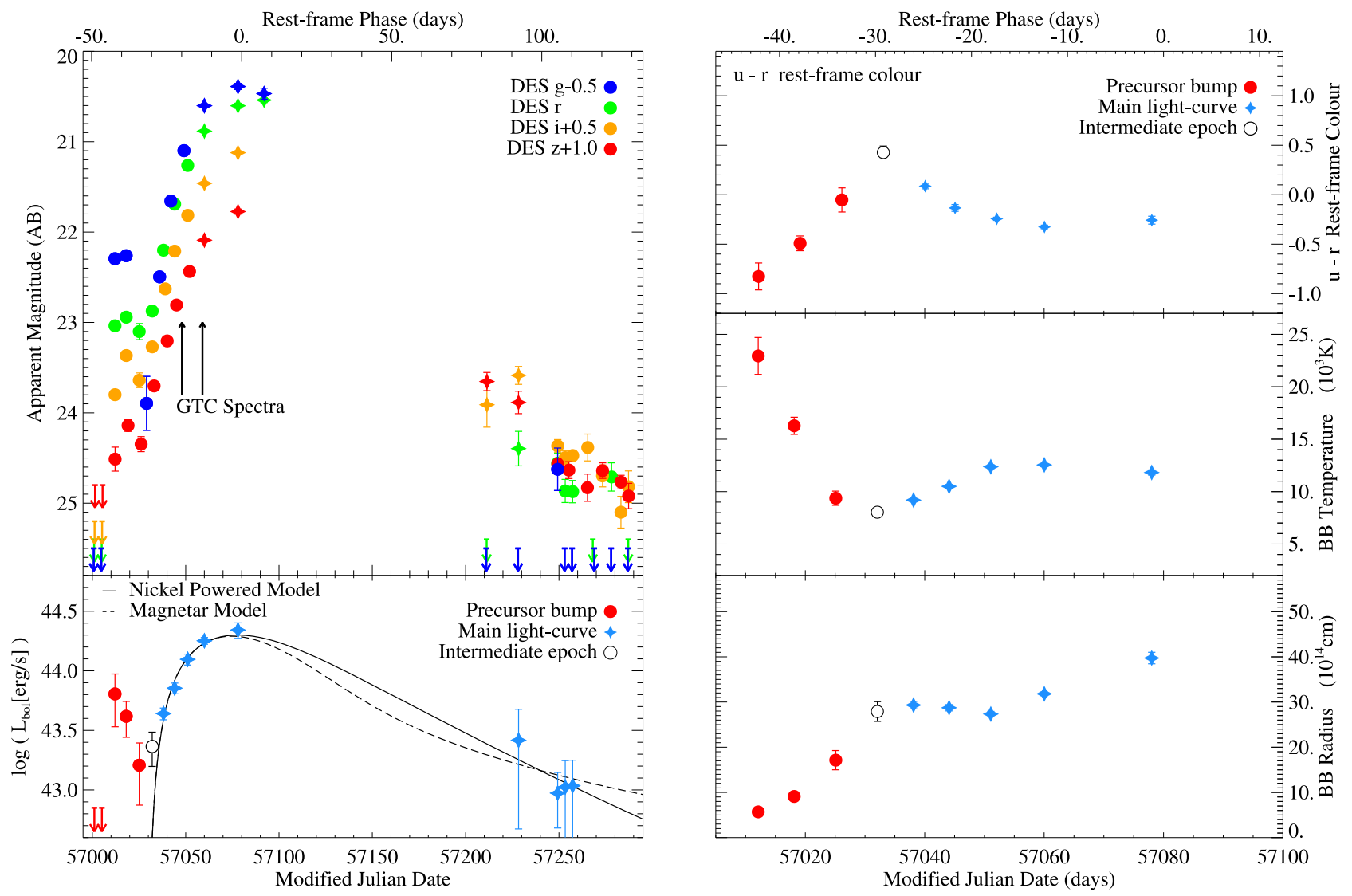

Figure 1. Photometric properties of DES14X3taz. Upper left: the multi-color observer-frame griz light curve from DES (circles) and SUDSS (crosses). The filters are offset for clarity. Upper limits are denoted with arrows, and the epochs of GTC spectroscopy are highlighted. Lower left: the bolometric light curve on epochs with three or more filter detections. Epochs associated with the initial peak are shown as red circles, with the main light curves plotted as blue crosses. Intermediate epochs are plotted as open circles. The main light curve has fits for both a ${ }^{56} \mathrm{Ni}$-powered model (solid; $M_{\mathrm{Ni}}=26 M_{\odot}$ ) and a magnetar spin-down-powered model (dashed; $\left.P_{\mathrm{ms}}=2.2 \mathrm{~ms}, B_{14}=1.3 \times 10^{14} \mathrm{G}\right)$. Upper right: the rest-frame $u-r$ color evolution. Middle and lower right: the evolution of the temperature and radius inferred from the blackbody fits to individual epochs of photometry. Details of $k$-corrections and blackbody fits are in Section 3.

at rest-frame 4200 and $4500 \AA$ are $\mathrm{O}$ II, seen in all SLSNe-I at this phase (e.g., Quimby et al. 2011; Inserra et al. 2013).

The host galaxy of DES14X3taz is detected in stacked images from DES that contain no SN light. Using SEXTRACTOR (Bertin \& Arnouts 1996), we measure host-galaxy AB magnitudes (MAG_AUTO) of $(g, r, i, z)=(26.16 \pm 0.39$, $25.07 \pm 0.13,24.95 \pm 0.13,25.00 \pm 0.18)$ after correcting for MW extinction. Using the Z-PEG photometric-redshift code (Le Borgne \& Rocca-Volmerange 2002), a Kroupa (2001) initial mass function, and the redshift fixed at $z=0.608$, we estimate a host-galaxy stellar mass of $\log \left(M / M_{\odot}\right)=8.0_{-0.2}^{+0.4}$ and a star formation rate of $\log (\mathrm{SFR})=-0.8_{-0.2}^{+0.6} M_{\odot} \mathrm{yr}^{-1}$. These properties are consistent with the hosts of other SLSNe (e.g., Neill et al. 2011; Leloudas et al. 2015; Papadopoulos et al. 2015).

\section{ANALYSIS}

We next study the unusual light curve of DES14X3taz. The most striking feature (Figure 1, upper left panel) is the presence of a first peak in the light curve around MJD 57015 (2014 December 24), prior to the main peak at MJD 57080 (2015 February 27). The initial peak is most pronounced in the bluer filters, but is still distinct in the $z$-band.

We estimate the rest-frame $u g r$ light curve from the observer-frame riz bands, which correspond to a similar wavelength range at $z=0.608$, using a standard $k$-correction procedure. With the limited wavelength coverage of our GTC spectra, pre- and at-max $k$-corrections are determined using the spectrally similar event LSQ14bdq, which has coverage down to $3600 \AA$ (Nicholl et al. 2015) and late-time $k$-corrections using data from PTF12dam at $+171 \mathrm{~d}$ (Nicholl et al. 2013). A polynomial fit to the rest-frame $g$-band light curve indicates DES14X3taz reached a peak brightness of $M_{g}=-21.39$ on MJD 57080 after correcting for foreground extinction and assuming zero internal reddening. We show the $u-r$ restframe color evolution in Figure 1 (upper right panel)-the initial peak is blue $(u-r \simeq-0.9)$ at first, but quickly reddens by nearly 1.5 mag to $u-r \simeq 0.5$ (before becoming bluer again during the main rise to peak brightness). Such reddening behavior is typical of a rapid decrease in temperature due to cooling.

We analyze this rapid evolution in more detail by fitting a blackbody to each epoch of photometry with observations of at least 3 filters within 4 days, using Planck's law and a luminosity distance of $3587 \mathrm{Mpc}$. (The epoch at MJD 57032 is excluded from these fits, as at this epoch there is potentially flux from both components of the light curve.) The evolution of the temperature and radius inferred from these fits is shown in Figure 1 (right panels) and demonstrates a rapid cooling from 22,000 to $8000 \mathrm{~K}$ over 15 rest-frame days. This is followed by a reheating phase during the main light curve, which then itself 


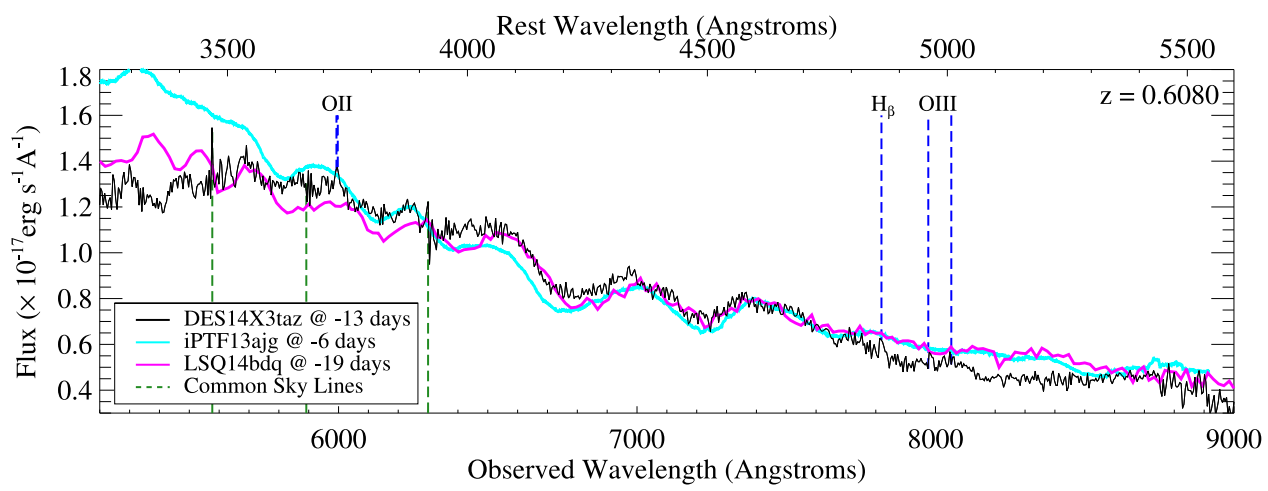

Figure 2. GTC optical spectrum of DES14X3taz from 2015 February 6 (black line). Galaxy emission features are highlighted in blue and prominent sky lines in green. Also shown are the spectra of the SLSNe-I iPTF13ajg at $-6 \mathrm{~d}$ (cyan; Vreeswijk et al. 2014) and LSQ14bdq at -19 d (magenta; Nicholl et al. 2015).

cools as it approaches the main peak, consistent with other literature on SLSNe-I.

The bolometric light curve (Figure 1, lower left panel) is also constructed by integrating the best-fit blackbody function on each epoch. The two peaks are particularly clear in bolometric luminosity: one, the initial peak, prior to MJD 57030; and the other, the main peak.

\subsection{The Main Peak}

We fit the bolometric light curve of the main peak with two models: energy deposition from the radioactive decay of ${ }^{56} \mathrm{Ni}$, and energy deposition from the spin-down of a rapidly rotating magnetar. In both cases, assuming a spherically symmetric and homologously expanding ejecta, the bolometric luminosity $L$ as a function of time $t$ since explosion is (Arnett 1982)

$$
L(t)=e^{-\left(t / \tau_{m}\right)^{2}} \int_{0}^{t} \frac{2 t^{\prime}}{\tau_{m}^{2}} P\left(t^{\prime}\right) e^{\left(t^{\prime} / \tau_{m}\right)^{2}} d t^{\prime},
$$

where $P(t)$ is the power function, the total absorbed power due to either ${ }^{56} \mathrm{Ni}$ or a central engine, and $\tau_{m}$ is the diffusion timescale parameter, a function of the ejecta mass $\left(M_{\mathrm{ej}}\right)$, opacity $(\kappa)$, and kinetic energy $\left(E_{k}\right)$. We assume a constant $\kappa=0.1 \mathrm{~cm}^{-2} \mathrm{~g}^{-1}$ (see the discussion in Inserra et al. 2013).

The power function appropriate for ${ }^{56} \mathrm{Ni}$ depends on the ${ }^{56} \mathrm{Ni}$ mass $\left(M_{\mathrm{Ni}}\right)$ synthesized in the explosion, $\tau_{m}$, and the time of explosion $t_{0}$ (e.g., Inserra et al. 2013; Papadopoulos et al. 2015). If we enforce $M_{\mathrm{ej}} \geqslant M_{\mathrm{Ni}}$, we obtain a ${ }^{56} \mathrm{Ni}$ mass of $M_{\mathrm{Ni}}=26 \pm 2.6 \quad M_{\odot}$ with $M_{\mathrm{ej}}=M_{\mathrm{Ni}}$ (shown in Figure 1). Although the fit is statistically acceptable, with a $\chi^{2}$ of 4.9 for 6 degrees of freedom (dof), such a model with $M_{\mathrm{ej}}=M_{\mathrm{Ni}}$ is difficult to reconcile with the observed spectra (see, e.g., the discussion in Inserra et al. 2013). Enforcing more physical constraints on the ratio of $M_{\mathrm{Ni}}$ to $M_{\mathrm{ej}}$ (e.g., Papadopoulos et al. 2015) then results in implied ejecta velocities significantly greater than observed in the spectra of SLSNe-I: for example, a limit of $M_{\mathrm{Ni}}<0.7 M_{\mathrm{ej}}$ requires velocities of $>22,000 \mathrm{~km} \mathrm{~s}^{-1}$, compared to $10,000 \mathrm{~km} \mathrm{~s}^{-1}$ measured from the DES14X3taz spectrum. Thus, like other SLSNe-I, the light curve of DES14X3taz cannot be fit with a physically plausible ${ }^{56} \mathrm{Ni}$ diffusion model.

The magnetar power function depends on two parameters. The initial spin period $\left(P_{\mathrm{ms}}\right.$, in milliseconds) and the initial magnetic field strength $\left(B_{14}\right.$, in $\left.10^{14} \mathrm{G}\right)$. Assuming full-trapping of the magnetar radiation, we find $P_{\mathrm{ms}}=2.2 \pm 1.4 \mathrm{~ms}$, $B_{14}=1.25 \pm 0.30 \times 10^{14} \mathrm{G}$, and $\tau_{m}=54.5 \mathrm{~d}$. From this, we infer an ejecta mass of $M_{\mathrm{ej}}=9.5 M_{\odot}$. The model fit has a $\chi^{2}$ of
5.4 with 5 dof. These values are similar to other SLSNe-I with initial peaks (e.g., LSQ14bdq: $P_{\mathrm{ms}}=1.7 \mathrm{~ms}$ and $B_{14}=$ $0.6 \times 10^{14} \mathrm{G}$; Nicholl et al. 2015) and SLSNe-I in general (e.g., DES13S2cmm: $P_{\mathrm{ms}}=5.3 \mathrm{~ms}$ and $B_{14}=1.4 \times 10^{14} \mathrm{G}$; Papadopoulos et al. 2015).

\subsection{Modeling the Initial Peak}

We next examine the initial peak in the light curve. We consider both a ${ }^{56} \mathrm{Ni}$ power source (for example, from an underlying $\mathrm{SN}$ explosion) and various shock-cooling models.

\subsection{1. ${ }^{56} \mathrm{Ni}$-powered Models}

As the main light curve is well modeled by a magnetardriven power source, it is appropriate to consider whether the initial peak in the light curve is consistent with a normal ${ }^{56} \mathrm{Ni}$ powered SN explosion, i.e., the SN that gave birth to the magnetar. We compare the observed multi-color light curve of the initial peak in DES14X3taz to various stripped envelope SNe (SESNe) from Bianco et al. (2014) and Modjaz et al. (2014). We use the templates of N. V. Karpenka et al. (2016, in preparation), where 28 events have been modeled using a simple parametric form allowing them to be placed at arbitrary redshifts (including $k$-corrections). As SLSNe-I (and DES14X3taz) are hydrogen-poor, we do not consider type II SNe.

None of the SESN templates are consistent with the initial peak in the DES14X3taz light curve: the initial peak rises faster, is significantly bluer at peak, and is $1.5 \mathrm{mag}$ brighter than any of the template events. Similarly, fitting the ${ }^{56} \mathrm{Ni}$ powered model from Section 3.1 to the initial peak requires $2.9 M_{\odot}$ of pure ${ }^{56} \mathrm{Ni}$ (i.e., $M_{\mathrm{Ni}}=M_{\mathrm{ej}}$ ) and a large inferred energy of $E_{k}=4.1 \times 10^{52} \mathrm{erg}$. Typical core-collapse events produce $\sim 0.1 M_{\odot}$ of ${ }^{56} \mathrm{Ni}$, and even a complete burning of $1 M_{\odot}$ of CO to ${ }^{56} \mathrm{Ni}$ produces only $1 \times 10^{51} \mathrm{erg}$ (Nicholl et al. 2015). Other power sources are clearly required.

\subsubsection{Shock Cooling of a Stellar Envelope}

We next consider the possibility that the initial peak of DES14X3taz is driven by shock cooling where, following an SN shock wave that heats the stellar envelope, the envelope expands and cools adiabatically, releasing energy. We use the analytical models of Rabinak \& Waxman (2011) and consider various progenitor envelopes: a radiative $\mathrm{H}$ envelope ("blue supergiant," BSG), a convective H envelope ("red supergiant," $\mathrm{RSG}$ ), He-dominated envelopes ("He envelopes") and C/O- 


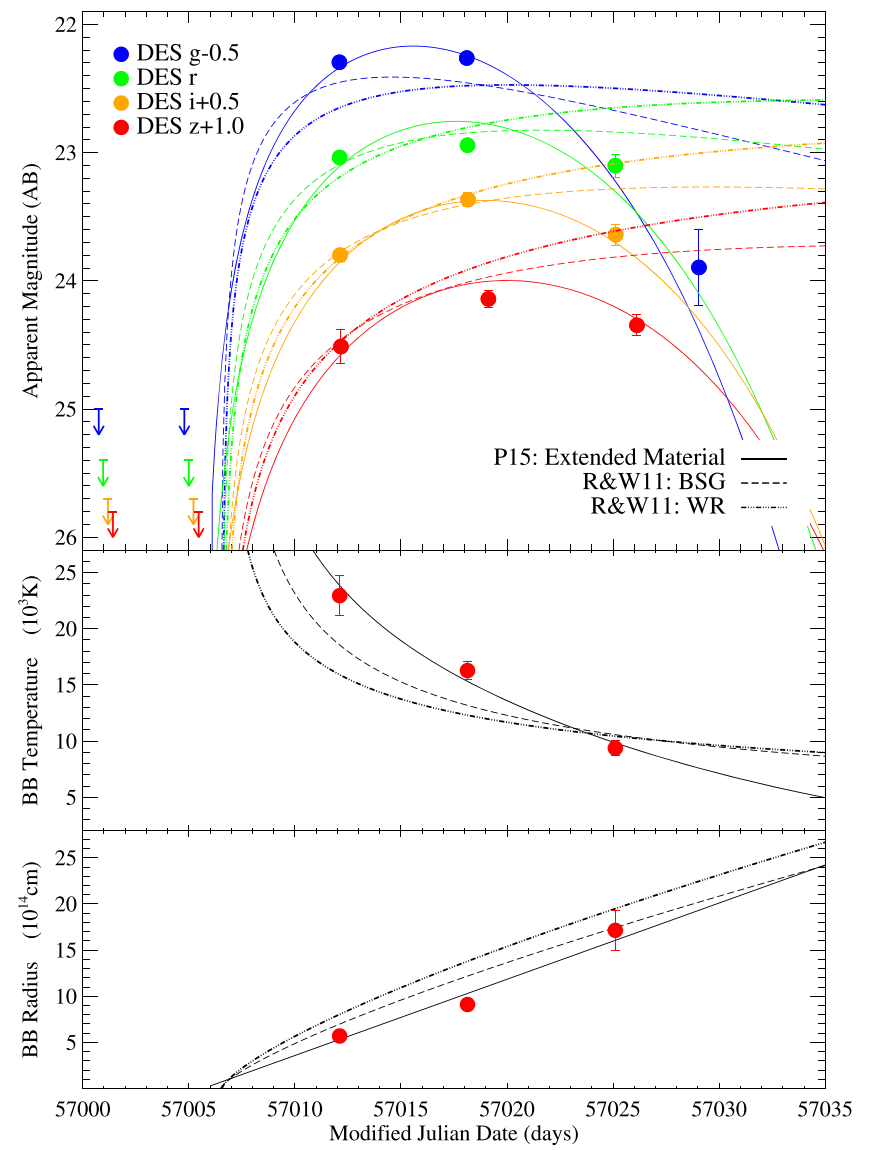

Figure 3. Top: various analytic models fit to the multi-color data of the initial peak of DES14X3taz. The best-fitting shock-cooling models from Rabinak \& Waxman (2011) of an extended envelope (BSG; dashed; $M_{\mathrm{ej}}=39 M_{\odot}$, $R_{\star}=530 R_{\odot}, E_{k}=1.5 \times 10^{52} \mathrm{erg}$ ) and a compact model (WR; dotteddashed; $M_{\mathrm{ej}}=500 M_{\odot}, R_{\star}=50 R_{\odot}, E_{k}=2.5 \times 10^{53} \mathrm{erg}$ ), together with an extended material model from Piro (2015; solid; $M_{\text {core }}=9.5 M_{\odot}, M_{\text {ext }}=2.7$ $\left.M_{\odot}, R_{\text {ext }}=400 R_{\odot}, E_{\mathrm{sn}}=6.0 \times 10^{51} \mathrm{erg}\right)$ are shown. Middle and lower panels: the DES14X3taz temperature and radius evolution from blackbody fitting (red circles; from Figure 1) compared to the shock-cooling models discussed above.

dominated envelopes ("Wolf-Rayet"). The Rabinak \& Waxman (2011) models are only valid at early times, so we cut off the models according to their Equation (17). We determine $L(t)$ from their relations and, assuming a blackbody, fit the models to the initial peak of DES14X3taz by determining fluxes in the DES bandpasses at $z=0.608$.

We show the results in Figure 3. The RSG, BSG, and He envelopes are very similar, so we only show the BSG example for clarity. Although the rise and color of the initial peak can be reasonably well matched by these models, in detail the models do not drop fast enough to match the DES14X3taz light curve and do not well reproduce the inferred temperature evolution, with a $\chi^{2}$ of 148 and 373 for the BSG and WolfRayet models, respectively (for 8 dof). The size of the envelopes implied are also very large (e.g., $\simeq 520 R_{\odot}$ for the BSG envelope and $\simeq 30 R_{\odot}$ for the C/O-dominated envelope). These are a factor of $\sim 10$ larger than typical BSGs or WolfRayet stars.

\subsubsection{Shock Cooling of Extended Material}

A similar model involves extended material around the progenitor star, but at much larger radii than the stellar

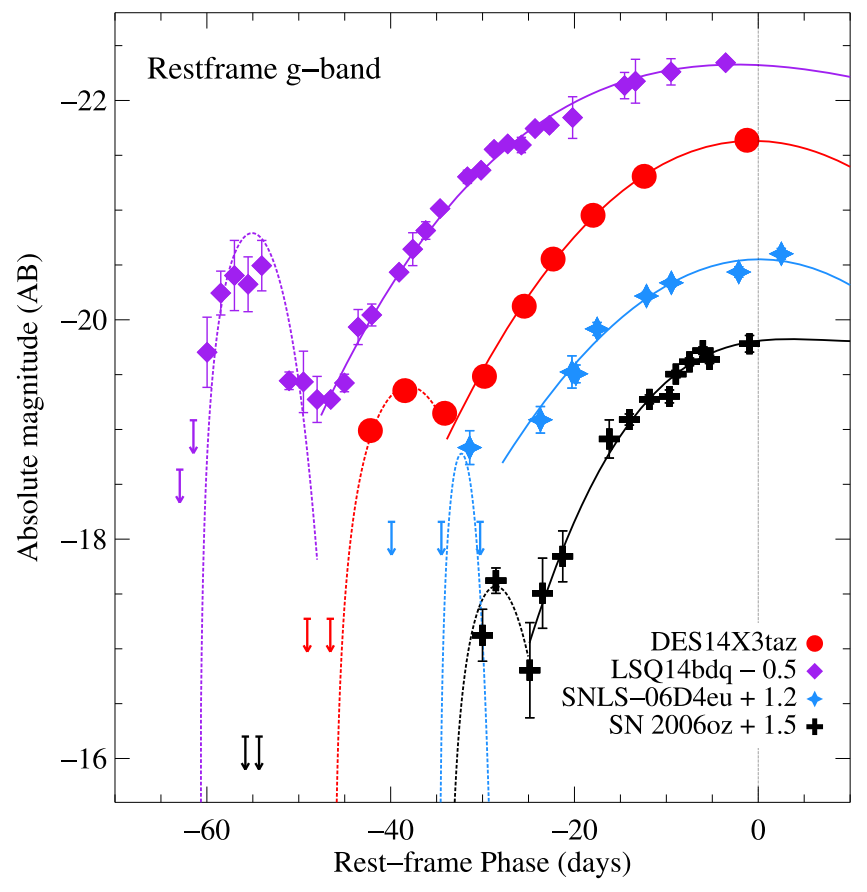

Figure 4. Rest-frame $g$-band light curve for DES14X3taz, compared to literature SLSNe-I with initial peaks. Offsets in absolute magnitude have been applied for clarity. Piro (2015) model fits are plotted over the initial peaks, and polynomial fits are overplotted on the main peak of each event. $3 \sigma$ nondetections are shown.

envelope. If this material is sufficiently massive and extended - perhaps from stripping or earlier pre-explosion eruptionsthe SN shock can propagate into it and produce a bright initial peak in the light curve (Ofek et al. 2010; Piro 2015) or, in the absence of further late-time energy input into the ejecta, an isolated bright, luminous transient (Drout et al. 2014).

We use the analytical relations of Piro (2015) to model this, showing the results in Figure 3. Due to degeneracies in the model between the core mass $\left(M_{\text {core }}\right)$ and energy, we fix $M_{\text {core }}=9.5 M_{\odot}$, the ejecta mass estimated from the magnetar fitting to the main peak (Section 3.1). The resultant fit has a $\chi^{2}$ of 45 for 9 dof, with $\simeq 2.7 M_{\odot}$ of material at a radius of $\simeq 400 R_{\odot}$ and an energy $E_{\mathrm{sn}}=6.0 \times 10^{51} \mathrm{erg}$. (Relaxing this $M_{\text {core }}$ constraint results in values of $M_{\text {core }}$ between 5 and $100 M_{\odot}$, but does not significantly alter the mass of extended material or the $\chi^{2}$.) This model reproduces the color, photometric evolution, and temperature evolution of DES14X3taz.

\section{DISCUSSION AND FUTURE WORK}

The discovery of double-peaked SLSNe-I provides new insights into the progenitors of SLSNe. The unique multi-color information of the initial peak of DES14X3taz reveals a very hot event that cools rapidly. We propose that this is driven by a period of shock cooling, with the SLSN phase of the light curve driven by reheating from a central engine, consistent with a magnetar. Shock cooling from an extended stellar envelope can match the color of the early part of the initial peak, but does not reproduce the fast dropoff, providing a poor overall match. By contrast, models with extended material at $\simeq 500 R_{\odot}$ can replicate the entire initial peak.

The quantity of data on double-peaked light curves of SLSNe-I is increasing quickly. In Figure 4, we compare the 
DES14X3taz rest-frame $g$-band light curve to other SLSNe-I from the literature that also exhibit initial peaks: SN2006oz $(z=0.376$; Leloudas et al. 2012), SNLS-06D4eu $(z=1.588$; Howell et al. 2013), and LSQ14bdq ( $z=0.345$; Nicholl et al. 2015). griz data of the initial peaks are available for SN2006oz (covering $\simeq 3400-6500 \AA$ rest-frame) and SNLS-06D4eu $(\simeq 1900-3400 \AA)$, but only data from a single broad " $g+r$ " filter are available for LSQ14bdq $(\simeq 4200 \AA)$.

Figure 4 shows diversity in the luminosity and duration of the initial peaks, especially when compared to the rise-time and peak magnitude of the resulting SLSN. However, only $\simeq 15$ literature SLSNe have well-measured pre-explosion photometry, at least $\simeq 30 \%$ of which have double peaks. Other initial peaks may be present in the remainder, but lie below the photometric detection thresholds of the discovery surveys. The initial peaks in Figure 4 are all consistent with the Piro (2015) model, perhaps suggesting that one general physical interpretation can explain the evolution of all SLSN-I events.

Understanding the initial peaks of SLSNe-I in more detail will require spectral (and therefore velocity and composition) measurements. Such measurements can be used to search for any hydrogen or helium signatures that may be present, if the model of extended material is indeed correct. These observations are technically challenging, as SLSNe are rare and would need to be identified rapidly from other SN types based on only 1-2 epochs of photometry. However, DES is expected to find $\simeq 5-6$ well-observed SLSNe-I per year (Scovacricchi et al. 2016), and the distinct properties of these SLSNe at early times (very blue colors, very faint host galaxies) allow them to be cleanly selected, making spectral coverage a realistic possibility in the near future.

We acknowledge support from EU/FP7-ERC grants 615929 and 307260, STFC, NSF grant AST-1518052, and a Weizmann-UK Grant. Based on observations made with the Gran Telescopio Canarias (GTC), at the Spanish Observatorio del Roque de los Muchachos of the Instituto de Astrofsica de Canarias.

Funding for the DES Projects has been provided by the DOE and NSF (USA), MEC/MICINN/MINECO (Spain), STFC (UK), HEFCE (UK), NCSA (UIUC), KICP (U. Chicago), CCAPP (Ohio State), MIFPA (Texas A\&M), CNPQ, FAPERJ, FINEP (Brazil), DFG (Germany), and the Collaborating Institutions in the Dark Energy Survey.

The Collaborating Institutions are Argonne Lab, UC Santa Cruz, University of Cambridge, CIEMAT-Madrid, University of Chicago, University College London, DES-Brazil Consortium, University of Edinburgh, ETH Zürich, Fermilab, University of Illinois, ICE (IEEC-CSIC), IFAE Barcelona, Lawrence Berkeley Lab, LMU München, and the associated Excellence Cluster universe, University of Michigan, NOAO, University of Nottingham, Ohio State University, University of Pennsylvania, University of Portsmouth, SLAC National Lab, Stanford University, University of Sussex, and Texas A\&M University.
The DES Data Management System is supported by the NSF under grant number AST-1138766. The DES participants from Spanish institutions are partially supported by MINECO under grants AYA2012-39559, ESP2013-48274, FPA2013-47986, and Centro de Excelencia Severo Ochoa SEV-2012-0234. Research leading to these results has received funding from the ERC including grants 240672, 291329, and 306478.

Facilities: Blanco, GTC.

\section{REFERENCES}

Arnett, W. D. 1982, ApJ, 253, 785

Benetti, S., Nicholl, M., Cappellaro, E., et al. 2014, MNRAS, 441, 289 Bertin, E., \& Arnouts, S. 1996, A\&AS, 117, 393

Bianco, F. B., Modjaz, M., Hicken, M., et al. 2014, ApJS, 213, 19 Chatzopoulos, E., Wheeler, J. C., \& Vinko, J. 2009, ApJ, 704, 1251 Chatzopoulos, E., Wheeler, J. C., Vinko, J., Horvath, Z. L., \& Nagy, A. 2013, ApJ, 773, 76

Chen, T.-W., Smartt, S. J., Bresolin, F., et al. 2013, ApJL, 763, L28

Chevalier, R. A., \& Irwin, C. M. 2011, ApJL, 729, L6

Chomiuk, L., Chornock, R., Soderberg, A. M., et al. 2011, ApJ, 743, 114

Dexter, J., \& Kasen, D. 2013, ApJ, 772, 30

Drout, M. R., Chornock, R., Soderberg, A. M., et al. 2014, ApJ, 794, 23

Firth, R. E., Sullivan, M., Gal-Yam, A., et al. 2015, MNRAS, 446, 3895

Flaugher, B., Diehl, H. T., Honscheid, K., et al. 2015, AJ, 150, 150

Gal-Yam, A. 2012, Sci, 337, 927

Gezari, S., Halpern, J. P., Grupe, D., et al. 2009, ApJ, 690, 1313

Goldstein, D. A., D’Andrea, C. B., Fischer, J. A., et al. 2015, AJ, 150, 82

Howell, D. A., Kasen, D., Lidman, C., et al. 2013, ApJ, 779, 98

Howell, D. A., Sullivan, M., Perrett, K., et al. 2005, ApJ, 634, 1190

Inserra, C., Smartt, S. J., Jerkstrand, A., et al. 2013, ApJ, 770, 128

Kasen, D., \& Bildsten, L. 2010, ApJ, 717, 245

Kasen, D., Metzger, B. D., \& Bildsten, L. 2015, arXiv:1507.03645

Kessler, R., Marriner, J., Childress, M., et al. 2015, AJ, 150, 172

Kroupa, P. 2001, MNRAS, 322, 231

Le Borgne, D., \& Rocca-Volmerange, B. 2002, A\&A, 386, 446

Leloudas, G., Chatzopoulos, E., Dilday, B., et al. 2012, A\&A, 541, A129

Leloudas, G., Schulze, S., Krühler, T., et al. 2015, MNRAS, 449, 917

Lunnan, R., Chornock, R., Berger, E., et al. 2013, ApJ, 771, 97

Lunnan, R., Chornock, R., Berger, E., et al. 2015, ApJ, 804, 90

McCrum, M., Smartt, S. J., Rest, A., et al. 2015, MNRAS, 448, 1206

Modjaz, M., Blondin, S., Kirshner, R. P., et al. 2014, AJ, 147, 99

Nakar, E., \& Sari, R. 2010, ApJ, 725, 904

Neill, J. D., Sullivan, M., Gal-Yam, A., et al. 2011, ApJ, 727, 15

Nicholl, M., \& Smartt, S. J. 2016, MNRAS, 457, 79

Nicholl, M., Smartt, S. J., Jerkstrand, A., et al. 2013, Natur, 502, 346

Nicholl, M., Smartt, S. J., Jerkstrand, A., et al. 2015, ApJL, 807, L18

Ofek, E. O., Cameron, P. B., Kasliwal, M. M., et al. 2007, ApJL, 659, L13

Ofek, E. O., Rabinak, I., Neill, J. D., et al. 2010, ApJ, 724, 1396

Papadopoulos, A., D’Andrea, C. B., Sullivan, M., et al. 2015, MNRAS, 449, 1215

Piro, A. L. 2015, ApJL, 808, L51

Quimby, R. M., Kulkarni, S. R., Kasliwal, M. M., et al. 2011, Natur, 474, 487

Quimby, R. M., Yuan, F., Akerlof, C., \& Wheeler, J. C. 2013, MNRAS, 431, 912

Rabinak, I., \& Waxman, E. 2011, ApJ, 728, 63

Scovacricchi, D., Nichol, R. C., Bacon, D., Sullivan, M., \& Prajs, S. 2016, MNRAS, 456, 1700

Smith, N., Li, W., Foley, R. J., et al. 2007, ApJ, 666, 1116

Vreeswijk, P. M., Savaglio, S., Gal-Yam, A., et al. 2014, ApJ, 797, 24

Woosley, S. E. 2010, ApJL, 719, L204

Yaron, O., \& Gal-Yam, A. 2012, PASP, 124, 668 